\title{
The Role that Self-Compassion and Self-Control play in Hostility provoked from a Negative Life Event
}

\author{
Richard H. Morley ${ }^{1}$, Victoria Terranova ${ }^{1}$, Shannon Cunningham ${ }^{1}$, Tyler Vaughn ${ }^{1}$
}

\section{ABSTRACT}

The main focus of this study is to investigate the degree to which self-compassion and selfcontrol buffer against hostility provoked by a negative life experience. To accomplish this inquiry pre and posttest state hostility measures were taken from sixty-six students displaying an increased negative affect following a statistics test. Repeat measures MANOVA revealed that the post measure increase in state hostility was significance $(\mathrm{p}>.05)$. Moreover, there was a significant interaction between self-compassion and self-control. Upon inspection of the group difference, participants with low self-compassion and low self-control score showed a significant increase on compared to pre-test scores or posttest scores compared to every other group. Correlational analysis revealed that while both variables were associated with pre and post-test measures of state hostility, Self-compassion was demonstrated to have a larger correlation than self-control. Moreover, the results, study limitations, and implications were discussed.

Keywords: Self-Compassion, Self-Control, Mindfulness, Aggression, Violence, Hostility, and Criminality.

Criminologists have connected the sources of violent criminality to undeveloped self-control, which is characterized by impulsive behavior, self-centeredness, increased propensity to engage in risky behavior, and a lack of anger control (Gottfredson \& Hirschi, 1990; Nagin \& Paternoster, 1993). Regardless of the theoretical origins, the intersection of impulsive behavior and negative life experience has been identified as provoking hostility, anger, and aggression.

In addition to risk factors of violence, many researchers are focusing on positive mental states that might reduce the risk of violent behavior (Woldgabreal, Day, \& Ward, 2014). One mental state that serves as a positive indicator of psychological wellbeing is self-compassion (Neff, 2003b). Furthermore, self-compassion is negatively associated neurological correlates associated with exposure to violence that have shown to predict anger, violence, and aggression (Klimecki,

\footnotetext{
${ }^{1}$ Texas State University, United States

*Responding Author

(C) 2016 I R Morley, V Terranova, S Cunninghan, T Vaughn; licensee IJIP. This is an Open Access Research distributed under the terms of the Creative Commons Attribution License (http://creativecommons.org/licenses/by/2.0), which permits unrestricted use, distribution, and reproduction in any Medium, provided the original work is properly cited.
} 
Leiberg, Lamm \& Singe, 2013; Longe et al., 2010). While research concerning the intersection of hostility and self-compassion is scarce, there have been many empirical affirmations to the value of the intersection of self-compassion and the risk of violence (Adams \& Leary, 2007; Neff \& Vonk, 2009; Web \& Forman, 2012).

\section{SELF-CONTROL THEORY}

Criminologists have recognized self-control, a construct similar to ASPD, as a theoretical cause of violence and criminality (Gottfredson \& Hirschi, 1990; Nagin \& Paternoster, 1993). Failure to develop self-control leads to a host of antisocial behaviors such asviolence. Self-control was described as "the extent to which they (people) are vulnerable to the temptations of the moment" (Gottfredson \& Hirschi, 1990, p.87), the inability to defer gratification (Nagin \& Paternoster, 1993), and the extent to which someone can adequately evaluate consequences of his/her behavior (Gottfredson \& Hirschi, 1990).

Gottfredson and Hirschi (1990) stated that low self-control is characterized by six elements. These factors include a lack of future orientation, self-centeredness, proneness to anger, lack of diligence, an orientation towards physical activities as compared to mental activities, and a preference for risk taking (Gottfredson \& Hirschi, 1990). This theory has been interpreted to suggest that a lack of self-control creates an inability to defer gratification (Nagin \& Paternoster, 1993). Similarly to ASPD, the underlying trait characterizing low self-control is impulsivity (Gottfredson \&Hirschi, 1990). Research studies have found that self-control was negatively associated with aggression (Netter, Hennig, Rohrmann, Wyhlidal, \& Hain-Hermann, 1998), the inability to defer gratification (Nagin \& Paternoster, 1993), psychopathy (Vaughn, DeLisi, Beaver, Wright, \& Howard, 2007), and violent re-offending (Grieger, Hosser, \& Schmidt, 2012; Piquero, MacDonald, Dobrin, Daigle, \& Cullen, 2005).

This section describes the link among exposure to violence, neurological correlates of violence, self-control, and the propensity to engage in violent behavior. Specifically, researchers theorize that exposure to violence creates neurological changes associated with impulsivity (Ducharme et al., 2011; Tow \& Whitty, 1955), psychopathy (Decety et al., 2013), reactive aggression (Damasio et al., 1994; Ducharme et al., 2011), and instrumental aggression (Blair, Colledge, Murray, \& Mitchell, 2001; Marsh et al., 2008). Furthermore, criminologists have conducted several studies that link low self-control, a construct similar to antisocial personality disorder, to psychopathy, impulsivity, aggression, and violent crime (Netter, Hennig, Rohrmann, Wyhlidal, \& Hain-Hermann, 1998; Grieger et al., 2012; Nagin \& Paternoster, 1993; Piquero et al., 2005; Vaughn et al., 2007).

\section{Self-Compassion}

Self-compassion is a concept characterized as a healthy self-attitude (Neff, 2003b). Selfcompassion involves the cognitive, affective, and behavioral self-dimensions of an experience and the motivation to enhance those dimensions through positive affect (Stosny, 1995). Stosny 
(1995) described self-compassion as an internal self-regulation of the perception of an experience that is "incompatible with antisocial behavior" (p. 82).

Neff conceptualized self-compassion as three components including self-kindness, mindfulness, and awareness of a common humanity (Neff, 2003a). Self-kindness refers to a propensity among people to show positive affect to themselves rather than being critical of oneself. Mindfulness involves being intentional and no judgmentally aware of one's personal thoughts and actions at the present (Grossman, Niemann, Schmidt, \& Walach, 2004; Neff, Hsieh, \& Dejitterat 2005). The final aspect of self-compassion concerns the recognition of a shared common humanity.

\section{Self-Compassion and Violence}

While little research directly connects self-compassion to violence, a few studies suggest that linking low self-compassion to violence shows promise. Neff and Vonk (2009) provided evidence that self-compassion was negatively associated with anger. Less anger perpetuates less aggression. Stosny (1995) developed the compassion workshop to increase self-compassion among crime perpetrators. He found that the participants who completed his program displayed more self-reported compassion as measured by the attachment compassion scale, less partner reported physical violence and less verbal violence (Stosny, 1995). The participants showed positive progress when compared to a cognitive behavioral intervention; a finding replicated with violent offenders by Murphy, Stosny and Morrel (2005).

While research has yet to establish a direct relationship between self-compassion and aggression, many studies have linked self-compassion to various predictors of violence. Specifically, evidence suggests that self-compassion is positively correlated with several variables shown to alleviate violence. These variables include: concern for others (Daugherty, Murphy, \& Paugh, 2001; Neff, 2003a), social connectedness (Neff, 2003b; Neff et al., 2007; Sampson \& Laub, 1993), and self-esteem (Murphy et al., 2005; Neff, 2003a; Oser, 2006). Moreover, preliminary evidence does suggest a link between self-compassion and different neurological structures associated with violence (Klimecki, Leiberg, Lamm \& Singe, 2013; Longe et al., 2010). Specifically, self-compassion seems to be associated with the insular cortex, the orbit frontal cortex, the striatum, the amygdala, and the anterior cingulate (Klimecki, Leiberg, Lamm \& Singer, 2012; Longe et al., 2010). Practicing self-compassion or engaging in compassion based meditation increases activation in areas deficient among violent persons, as well as decreasing areas overactive among perpetrators of aggression.

\section{Self-Compassion and Self-Control}

While little research has investigated the relationship between self-compassion and self-control, there seems to be a few key points of intersection. For instance Mindfulness, a previously mentioned subcomponent of self-compassion, shares many similarities to self-control (Bowlin \& Baer, 2012; Masicampo \& Baumeister, 2007). Specifically, mindfulness relates to the awareness of thoughts and feelings at the present moment while self-control has been described as 
susceptibilities to "temptations of the moment" (Gottfredson \& Hirschi, 1990, p.87). Greater self-awareness of a person's thoughts and actions could mean more control over one's susceptibilities. While similar, there are certain theoretical distinctions between the two traits. Mindfulness, specifically, is a dynamic trait while self-control is theorized to be invariant (Gottfredson \& Hirschi, 1990).

In addition to these intersections, researchers have discussed potential theoretical links between these two concepts. Neff (2003b) stated that self-compassion requires met cognitive skills, which require a certain level of self-control. Evidence shows that positive changes in mindfulness can lead to changes in self-compassion among university students (Bergen-Cico, Possemato, \& Cheon, 2013). As mentioned above, mindfulness involves self-control and is antagonistic to impulsivity. Under this assumption, self-control could be the cognitive structure required to have self-compassion.

While some evidence indicates that a certain level self-control is required for self-compassion, other findings indicate that self-compassion can be tied to decreases in impulsivity. Specifically, Heatherton and Baumeister (1991) theorized that self-awareness leads to an unpleasant view of the self, and impulsivity is linked to the avoidance of self-awareness. They later argue that selfregulation, exhibited through a lack of impulsivity, varies across person and across type of task (Heatherton and Baumeister, 1996). This theory has been supported in a variety of studies examining the relationship between eating disorders and self-compassion (Adams \& Leary, 2007, Web \& Forman, 2013).

In order to evaluate the concept that self-control and self-compassion are associated with violence, this study seeks to investigate the role that these characteristics play in changes in an individual's state hostility provoked from the context of a negative life experience. Specifically, this study will compare changes in hostility among 66 students displaying an increased negative affect in response to a statistics exam. Statistics can be a difficult and frustrating subject for many students and exams tend to amplify feelings of frustration. Considering that examination grades are relevant to the students' lives, this design captures change in hostility in the context of a real world event.

\section{METHODS}

\section{Procedure}

Participants were undergraduate students enrolled in a statistics course. In order to preserve the students' anonymity, the surveys did not include any demographic information other than gender. However, it is expected that due to the demographics of the classroom the participants were mostly white non-Hispanic and white Hispanic.

Students were surveyed before taking and after receiving their grade on a statistics test. Research assistants recruited students at the beginning of the lecture. In order to maintain anonymity, the 
instructor was not present while the participating students were surveyed. Students were asked to draw a symbol and number on the envelopes of both surveys so that researchers were able to connect the items from both tests without knowing the identity of the participants. The pretests were delivered two weeks prior to a statistics test. During the pretest, students completed a gender demographics form, the self-control scale, the PANAS, the shortened self-compassion form, and a hostility scale. Posttest surveys were taken immediately after the test scores were released. Test grades were posted two days prior to the posttest survey. Prior to distributing the posttest surveys, students were asked to look at their grades within their convenience within this time frame. Students who reported a negative life experience were included in the final analyses involving the impact of self-control and self-compassion on hostility. This included 66 participants including 32 men and 34 women.

\section{Measures}

Demographics. The demographic form included a question requesting gender information. The posttest demographic questionnaire included an item eliciting the students' evaluation of their test performance.

The Self-Compassion Short Form scale. The Self-Compassion short form is a 12-item selfreport instrument scale. It contains six subscales including three positive scales and three negative scales. These include the following: Self-kindness (e.g., I try to be understanding and patient towards those aspects of my personality I don’t like), Common Humanity (e.g., I try to see my failings as part of the human condition), and Mindfulness (e.g., When something upsets me, I try to keep my emotions in balance). Further, it contains the following three negative subscales: Self-judgment (e.g., I'm disapproving and judgmental about my own flaws and inadequacies), Isolation (e.g., When I fail at something important to me, I tend to feel alone), and Over-identification (e.g., When I'm feeling down, I tend to obsess and fixate on everything that's wrong).

Negative items (self-judgment, isolation, and over-identification) are reverse scored prior to completing the total mean. This measure has been shown to have good validity and reliability (Raes, Pommier, Neff \& Gucht, 2011) In this study, this measure yielded a Cronbach's alpha of .84 .

Positive and Negative Affect Schedule (PANAS).To measure the participants' affective states, this study used the Positive and Negative Affect Schedule (PANAS).Watson et al. (1988) developed this inventory of positive (such as determined, proud, enthusiastic) and negative emotions (such as nervous, guilty, upset). Items are rated based off of the participants' feelings at the present moment from "not at all” to “extremely."The pre test and post test measures yielded Cronbach’s alpha of .90 and .92 respectively.

Self-Control. To measure self-control in this study, Grasmick et al.'s (1993) self-control scale was used. Grasmick et al.’s (1993) Self-Control Scale has been the most widely used measure of 
self-control in the literature (Gibson, 2005). This scale was designed to measure self-control as defined by Gottfredson and Hirschi (1990), who described a lack of self-control as having six components. These six parts include: impulsivity (e.g., I often act on the spur of the moment without thinking), a preference for simple rather than complex tasks (e.g., I try to avoid projects I think are difficult), risk-seeking behavior (e.g., I test myself now and then by doing something risky), a preference for physical activities over cerebral activities (e.g., I like to get out and do things more than I like to read or contemplate ideas), self-centered orientation (e.g., I try to look after myself even if it means making things difficult for other people), and anger control (e.g., I lose my temper pretty easy). This 24-item scale includes 4 items for each component. These items are rated on a five-point Likert scale: never (0), rarely (1), sometimes (2), often (3), and almost always (4). Seven of the items are reverse coded. Higher scores on this scale indicate a lower level of self-control. This measurement was shown to have high reliability (.87) for incarcerated men (Gibson, 2005).In this study Cronbach’s alpha was found .85.

State Hostility Scale. In order to measure the participant's propensity for reactive aggression, they completed the state hostility scale (SHS)(Anderson, Deuser, \& DeNeve, 1995). The SHS is a 35-item Likert scale measure, which consist of statements concerning current emotions including 24 items related to anger (such as "I feel aggravated" and "I feel like yelling”) and 11 negatively scored pro-social emotional states (such as “I feel calm” and “I feel cooperative”). The SHS has demonstrated good internal reliability estimated from (Anderson, Deuser, \& DeNeve, 1995). Chronbach's alpha for the pretest and the posttest were .94 and .93 respectively.

Exam Satisfaction. Exam satisfaction was measured using a one-item Likert scale "How happy were you with your grade?” This item was rated on a four-point scale (4) strongly agree, (3) agree, (2) disagree, (1) and strongly disagree.

Negative Life Experience. In order to determine if a participant suffered from a negative life experience, every participant's posttest negative affect score was subtracted from their pretest score. Participants that divided based upon the polarity of their scores. Those that reported a lower pretest scored where coded as having a negative life experience and those that reported a larger pretest score were coded as having a positive one. Participants that received a negative number were recruited for further analyses. These participants included 32 males and 34 females for a total of 66 participants.

\section{RESULTS}

The initial validation analyses involved correlational analyses to evaluate to the degree to which the negative life experience variable correlated with exam satisfaction as well as pre and posttest negative affect. The analyzes revealed that the negative experience was negatively correlated with exam satisfaction $(\mathrm{R}=-.9 \mathrm{p}<.05)$ but positively correlated with negative affect $(\mathrm{R}=.69$ $\mathrm{p}<.05)$. Negative experience was not correlated with pretest negative affect. 
The analyses consisted of two-way repeat measures MANOVA and correlational analyses. Prior to testing assumptions, the pre- and posttest mean were calculated for both the self-control scores and self-compassion scores. In order to measure the impact of self-control and self-compassion on state anger, participants were separated in groups based off of their self-control and selfcompassion scores. Prior to splitting each group, Kolmogorov-Simon test was conducted concerning self-compassion and self-control scores. Kolmogorov-Simon test revealed that selfcompassion and self-control violated the normality assumption (.121 and .126 p<.05). Due to this violation, groups were assigned based off of percentiles rather than standard deviations. The split was determined based on the 33 and 66 percentile. Specifically, subjects who scored up to the 33 percentile were placed in the low self-compassion or self-control group, 34 to 66 percentile were placed in the medium self-control or self-compassion group, and participants over the 66 percentile were placed in the high self-control or self-compassion group.

Prior to conducting the two-way repeat measures MANOVA, each assumption was tested. Our Box's Test of Equality of Covariance Matrix showed an inequality of covariance (F 21, 3729.23= 1.88, $\mathrm{P}<.05$ ). Further, Levene's Test of Equality of Error Variance revealed that the pretest $(\mathrm{F}(8,55)=3.36 \mathrm{p}<.05)$. ANOVA's are robust to violations of the homogeneity of variance assumption (Lindman, 1974). Therefore, there were no special modifications made to these analyses. Moreover, this study used Kolmogorov-Simon test to revealed a violation of normality assumption on the pre and posttest hostility scores (.144 and .163, $<<.05)$. As with the homogeneity assumption, ANOVA is robust to violations of normality (Glass, Peckham, \& Sanders, 1972).

The two-way repeat Measure ANOVA was used to compare the effect of the self-compassion variable and the self-control variable on the state anger means differences between pre- and posttest. In terms of pre- and posttest scores, the Two Way Repeat Measures ANOVA revealed that the post-test hostility scores were significantly different from the pretest scores $\mathrm{F}(1,55)=$ $4.30 \mathrm{p}<.05 \eta 2=.07$ ) with a medium effect size (Cohen, 1977). As indicated by table 1 the posttest mean hostility scores were significantly larger than the pre-test.

Further, there were no differences found between either the self-compassion groups $(\mathrm{F}(2,55)=$ $.213 \mathrm{p}>.05)$ or the self-control groups $(\mathrm{F}(2,55)=1.22 \mathrm{p}>.05)$. However, there was an interaction between the self-compassion and the control groups $(\mathrm{F}(4,55)=4.82 \mathrm{p}<.05 \eta 2=.26)$ with a large effect size (Cohen, 1977). As indicated in table 1, participants within both the low self-control group and the low self-compassion group scored a sample mean of 122 on the posttest. Moreover, the estimate of the confidence intervals $(107.05,137.7)$ of participants in both these groups indicate that results of the participants are $95 \%$ more likely to score higher on the hostility score on the post-test than their pretest mean score (85.25). The same is true for the grand mean (75.47) or any other groups’ post-test score (55.5 to 81.45).

In addition to the Two-way Repeat Measures MANOVA, correlational analyses were implemented. As indicated on table 2, these findings suggest students' posttest scores were significantly associated with pretest scores $\mathrm{R}=.51 \mathrm{p}<.05$, self-compassion $\mathrm{R}=-.49 \mathrm{p}<.05$, and self- 
control $\mathrm{R}=.33 \mathrm{p}<.05$. The pretest hostility score displayed a significant correlation with selfcompassion $\mathrm{R}=-.56 \mathrm{p}<.05$ and self-control $\mathrm{R}=.30 \mathrm{p}<.05$. In addition, self-compassion was correlated with self-control $\mathrm{R}=-.25 \mathrm{p}<.05$.

\section{DISCUSSION}

The goal of this study was to investigate the role of self-control and self-compassion in predicting hostility in response to a negative life event. To accomplish this task, students were surveyed who displayed a negative change in affect in response to a statistics test. The preliminary analyses revealed the negative life event was valid in that it displayed a strong $(\mathrm{R}=.9)$ negative relationship with exam satisfaction, a strong positive relationship with posttest negative affect, and no relationship with pretest negative affect. Our sequent observationsindicateda interaction involving self-control and self-compassion. Moreover, these findings demonstrate that both self-compassion and self-control were correlated with state hostility during the pre- and post-test periods. Interestingly, the self-compassion score revealed a stronger correlation coefficient with the pre- and post-test hostility measures. Self-control scores displayed moderate correlation with both measures while self-compassion was found to have a large relationship than self-control (Cohen, 1988). In addition, self-control and self-compassion were correlated.

As mentioned above, the interaction among variables is most likely due to the significant increase in state hostility scores found among the participant with low self-control and low selfcompassion. Based off the MANOVA, neither self-compassion nor self-control as a particular group displayed an independent impact on state hostility. Rather, it was the combination of the two variables. These findings suggest that neither variable by itself impacts the tendency to become hostile after an adverse life event and that the low scores on both variables predict an increased state hostility.

Before discussing why the participants with low self-control and low self-compassion displayed significant increases in self-reported hostile tendencies, it is important to discuss potentialities as to why not having neither low self-control nor self-compassion could affect hostility. For instance, why did the group with moderate self-control but low self-compassion not show the same magnitude of increased state anger? A person with moderate levels of self-control but low self-compassion would be susceptible to self-criticism. While a person meeting these criteria would be vulnerable to internalizing negative life events, they would possess the met cognitive fortitude to resist hostile tendencies. These individuals may be likely to redirect blame inward by over-identifying (Neff 2003b) with their problems rather than projecting them.

Another possibility is that these individuals might restrain violent affect due to possible social sanctions that are reserved by society for those in the community who display overt acts of anger or aggression. With the legal or social penalties attached to displays of hostile behavior, these individuals may be deterred from hostile behavior by a rational calculation of the social costs of this type of behavior. In this case, a low self-compassion/ moderate self-control person would 
operate within the cognitive framework of the rational choice theory. In terms of neurological structures related to emotional regulation, people fitting this description are likely to have problems associated with the structures associated with self-criticism such as the insular cortex (Longe et al. 2010) but they have functional structures associated with self-regulation such as the orbit frontal cortex.

For the participants who displayed low levels of self-control and moderate levels of selfcompassion, a lower susceptibility to hostility is likely to involve the affective perspective of the individual rather than a met cognition. Specifically, low self-control/ moderate self-compassion individuals likely have lower inhibitions than those with low self-compassion/ high self-control. If faced with adverse life events, these individuals are likely to have a diminished ability to regulate negative feelings that occur. Rather, their cognitive appraisal of the adverse event provokes less of an emotional response. Perhaps these individuals respond to adverse life events with compassion rather than hostility. Considering Nagin and Paternoster's (1993) description of low self-control as the inability to defer gratification, perhaps individuals who are selfcompassionate gain less gratification from hostile actions. Possibly these participants might respond to negative life events with more self-kindness. Perhaps these individuals are mindful of the impulsive behavior and use self-compassion as an adaptive measure to prevent hostile tendencies.

For the participants in this study that had low self-control and low self-compassion, these findings indicate a tendency to respond in a hostile manner to adverse life events. Participants who fall in this category may have impaired self-regulation and the negative affective perspective of the event. Due to the combination of negative appraisal and a lack of selfregulation, negative events are likely to provoke negative emotions including anger and aggression. Moreover, individuals with low self-compassion and self-control are unable to look at themselves and more likely to externalize their blame in the form of aggression (Stosny, 1995). People with low self-control and self-compassion would probably demonstrate abnormalities in brain regions associated with self-regulation and emotional regulation (Baerentsen, Hartvig, Stodkilde-Jorgensen, \& Mammen, 2001; Cahn \& Polich, 2006, Hölzel et al., 2007; Holzel et al., 2011; Klimecki et al., 2012; Lazar et al., 2003; Longe et al., 2010; Wang et al., 2011). These areas of the brainhave been found to be associated with hostility, violence, criminality, and antisocial behavior (Aoki, Inokuchi, Nakao, \& Yamasue, 2013; Damasio et al., 1994; Moffit, 1993; Schiffer et al., 2014).

This study also identifies the magnitude of the difference between the correlation coefficient of the two independent variables. Specifically, self-compassion shared a high negative correlation with the reactive hostility measure before and after the adverse life event, while self-control only exhibited a moderate correlation at both times. A likely rationale for the super efficacy of selfcompassion is that self-compassion is specific to negative experience (Neff \& Dahm, 2014) while self-control is applicable to any life event. 
While these results demonstrate the superior efficacy of self-compassion compared to selfcontrol as a predictor of hostility, self-control is still an important factor. As indicated by these MANOVA results, participants only displayed significant increases in state anger when there was a lack of both traits. Furthermore, self-control did reveal a moderate relationship with state anger. This is consistent with the literature pertaining to self-control and violence, which establishes a link between aggressive tendencies and self-control (Grieger et al., 2012; Nagin \& Paternoster, 1993; Netter et al., 1998; Piquero et al., 2005; Vaughn et al., 2007).

\section{RESEARCH IMPLICATIONS}

While the findings in this study were promising, the research connecting self-compassion to selfcontrol and violence is mostly theoretical. While hostility is intrinsically connected to violence, people can have hostile feelings without aggressing. Future studies will have to examine the role a lack of self-compassion plays in violence and criminality specifically. One question that seems to arise from these results relates to the different mechanisms as well as brain regions involving self-control and self-compassion as they relate to anger management. This paper theorizes that self-control has more to do with self-regulation and self-compassion to do with perspective. This introduces the potential need for examinations of the different mechanisms involved in abating hostile feelings. Researchers could investigate the neurological structures involved in self-control versus self-compassion during the process of emotional control. Considering there is much overlap between these two areas, these two variables are likely to involve similar networks. Because of this a causal connection may be established between self-control and selfcompassion. Perhaps, future study could apply an intervention designed to change one variable and measure if this change creates correlated changes on the other variable.

Further study should also include examination of the extent that self-compassion may predict criminal recidivism. Identification and further description of the relationship between selfcompassion and re-offending could inform criminal justice agency intervention practices. A final direction that could be examined relates to the degree to which these variables, selfcompassion and self-control, impact participants' response to violent media. A significant amount of research literature suggests a strong connection between exposure to violence and aggression. If these variables diminish changes in aggression associated with exposure to violence then, they will make a useful therapeutic tool.

\section{CLINICAL IMPLICATIONS}

While research investigating the use of self-compassion, as an intervention in violence is limited, these findings along with other research studies suggest that integrating self-compassion may have therapeutic value. One program, the compassion workshop, targets increases in selfcompassion to reduce violence among perpetrators of domestic violence (Stosny, 1995). Including other types of violent offenders beyond domestic to allow for treatment of selfcompassion for a variety of violent behavior. Another potential clinical application might be to incorporate mindfulness based meditation and loving kindness meditation into existing 
interventions. Mindfulness-based meditation and loving kindness meditation have been shown to increase self-compassion (Birnie, Speca, \& Carlson, 2010; Kuyken et al., 2010; Logie \& Frewen, 2014). Development a curriculum that targets increasing the self-compassion of at-risk youths could have positive implications on addressing youth violence. Finally, another application could be to target positive increases in self-compassion in violence prevention and anger management programs.

\section{LIMITATION}

This study had many limitations including the use of self-report scales, the inability to link the change in affect directly to the test, the lack of demographic information, and the absence of behavioral data. It is worth noting that changes in state hostility were measured using self-report. Self-report is susceptible to bias and fabrication (Howard \& Daily, 1979;Podsakoff, MacKenzie, Lee, \& Podsakoff, 2003). While the study's survey did not provide any direct reason for participant false reporting, such a risk is not impossible.

Without observed behavior, a direct causal relationship between the study variables and displays of hostility cannot be defined. In addition, the validity of the independent variables relies on a negative change in self-reported emotional scales temporally associated with the stimulus, the statistics exam. It is beyond the framework of this study to determine if a change of emotions was caused by the stimulus rather than events outside the study. A final limitation of this study involves lack of demographic information. There are potential differences due to culture, race, and ethnicity and self-compassion that are not addressed in this study. These differences may be of significance to the validity of study findings because they may contribute to covert bias, unidentified in the models.

In conclusion, these results suggest that self-compassion shows a moderate, positive relationship with self-control, a strong correlation with state anger prior to, and a strong association with state anger after experiencing an adverse life event. In addition, these findings indicate that a lack of self-compassion and low self-control display a greater propensity to hostile feelings after a subject is exposed to adverse life events. Overall, these results provide strong evidence that selfcompassion provides a defense against anger in response to a negative life circumstance.

\section{REFERENCE:}

Adams, C. E., \& Leary, M. R. (2007). Promoting self-compassionate attitudes toward eating among restrictive and guilty eaters. Journal of Social and Clinical Psychology, 26: 11201144.

American Psychiatric Association. (1994). DSM 4. American Psychiatric Association

American Psychiatric Association. (2000) Diagnostic and Statistical Manual-Text Revision (DSM-IV-TRim, 2000). American Psychiatric Association.

American Psychiatric Association. (2013). DSM 5. American Psychiatric Association. 
Anderson, C. A., \& Bushman, B. J. (2001). Effects of violent video games on aggressive behavior, aggressive cognition, aggressive affect, physiological arousal, and prosocial behavior: A meta-analytic review of the scientific literature. Psychological science, 12(5), 353-359. doi:10.1111/1467-9280.00366

Anderson, C. A., Deuser, W. E., \& DeNeve, K. M. (1995). Hot temperatures, hostile affect, hostile cognition, and arousal: Tests of a general model of affective aggression. Personality and Social Psychology Bulletin, 21, 434-448

Aoki, Y., Inokuchi, R., Nakao, T., \& Yamasue, H. (2013). Neural bases of antisocial behavior: a voxel-based meta-analysis Running title: Antisocial brain: a meta-analysis of VBM. Social cognitive and affective neuroscience, nst104. doi: 10.1093/scan/nst104

Baerentsen, K. B., Hartvig, N. V., Stødkilde-Jørgensen, H., \& Mammen, J. (2001). Onset of meditation explored with fMRI. Neuroimage, 13(6), 297.*

Bergen-Cico, D., Possemato, K., \& Cheon, S. (2013). Examining the Efficacy of a Brief Mindfulness-Based Stress Reduction (Brief MBSR) Program on Psychological Health. Journal of American College Health, 61: 348-360.

Birnie, K., Speca, M., Carlson, L. E. (2010). Exploring self-compassion and empathy in the context of Mindfulness-based Stress Reduction (MBSR). Stress and Health, 26, 359-371

Blair R.J., Colledge E., Murray L., \& Mitchell D.G. (2001)A selective impairment in the processing of sad and fearful expressions in children with psychopathic tendencies. J Abnorm Child Psychol.;29(6):491-498. doi: 10.1023/A:1012225108281

Bowlin, S. L. \& Baer, R. A. (2012) Relationship between mindfulness, self-control and selfregulation. Journal of Personality and Individual Differences, 52: 411-515

Burt, C.H., Simons, R.L., \& Simons, LG. (2006). A longitudinal test of the effects of parenting and the stability of self-control: Negative evidence for the general theory of crime. Criminology, 44(2), 353-396.

Cahn, B. R., \& Polich, J. (2006). Meditation states and traits: EEG, ERP, and neuroimaging studies. Psychological bulletin, 132(2), 180. doi: 10.1037/0033-2909.132.2.180

Cohen, J. (1988). Statistical power analysis for the behavioral sciences (2nd ed.). Hillsdale, NJ: Lawrence Earlbaum Associates.

Crawford, J. R., \& Henry, J. D. (2004). The Positive and Negative Affect Schedule (PANAS): Construct validity, measurement properties and normative data in a large non-clinical sample. British Journal of Clinical Psychology, 43(3), 245-265.

Damasio H, Grabowski T, Frank R, Galaburda AM, Damasio AR. (1994). The return of Phineas Gage: Clues about the brain from the skull of a famous patient. Science 264:1102-1105.

Daugherty, D. A., Murphy, M. J., \& Paugh, J. (2001). An examination of the Adlerian construct of social interest with criminal offenders. Journal of Counseling \& Development, 79(4), 465-471. doi: 10.1002/j.1556-6676.2001.tb01994.x

De Bellis, M. D. (2005). The psychobiology of neglect. Child Maltreatment, 10(2), 150-172. doi: $10.1177 / 1077559505275116$ 
Decety, J., Chen, C., Harenski, C., \& Kiehl, K. A. (2013). An fMRI study of affective perspective taking in individuals with psychopathy: imagining another in pain does not evoke empathy. Frontiers in human neuroscience, 7. doi: 10.3389/fnhum.2013.00489

Ducharme, S., Hudziak, J. J., Botteron, K. N., Ganjavi, H., Lepage, C., Collins, D. L., ... \& Karama, S. (2011). Right anterior cingulate cortical thickness and bilateral striatal volume correlate with child behavior checklist aggressive behavior scores in healthy children. Biological psychiatry, 70(3), 283-290. 10.1016/j.biopsych.2011.03.015

Engström, M., \& Söderfeldt, B. (2010). Brain activation during compassion meditation: a case study. The Journal of Alternative and Complementary Medicine, 16(5), 597-599. doi:10.1089/acm.2009.0309.

Gibson, C. L. (2005). A psychometric investigation of a self-control scale: The reliability and validity of Grasmick et al.'s scale for a sample of incarcerated male offenders, Unpublished Dissertation, University of Nebraska

Gottfredson, H. G., \& Hirschi, T. (1990) A general theory of crime. Stanford, CA: Stanford University Press.

Grasmick, H. G., Tittle, C. R., Bursik, R. J., \& Arneklev, B. J. (1993). Testing the core empirical implications of Gottfredson and Hirschi's general theory of crime.Journal of research in crime and delinquency. 30(1): 5-29.

Grieger, L., Hosser, D., \& Schmidt, A. F. (2012). Predictive validity of self-reported self-control for different forms of recidivism. Journal of Criminal Psychology, 2: 80-95.

Grossman, P., Niemann, L., Schmidt, S., \& Walach, H. (2004). Mindfulness-based stress reduction and health benefits: A meta-analysis. Journal of psychosomatic research, 57(1), 35-43. doi: 10.1016/S0022-3999(03)00573-7

Hart, H., \& Rubia, K. (2012). Neuroimaging of child abuse: a critical review. Frontiers in human neuroscience, 6.doi: 10.3389/fnhum.2012.00052

Hay, C. \& Forrest, W. (2006). The development of self-control: Examining self-control theory's stability thesis. Criminology, 44(4), 739-774.

Heatherton, T. F., \& Baumeister, R. F. (1991). Binge eating as escape from self-awareness. Psychological bulletin, 110: 86-108

Heatherton, T.F. \& Baumeister, R.F. (1996). Self-regulation failure: Past, present, and future. Psychology Inquiry. 7(1): 90-98.

Hirschi, T., \& Gottfredson, M. R. (1995). Control theory and the life-course perspective. Studies on Crime \& Crime Prevention. 4(2): 131-142.

Hölzel, B. K., Lazar, S. W., Gard, T., Schuman-Olivier, Z., Vago, D. R., \& Ott, U. (2011). How does mindfulness meditation work? Proposing mechanisms of action from a conceptual and neural perspective. Perspectives on Psychological Science, 6(6), 537-559. doi: $10.1177 / 1745691611419671$

Hölzel, B. K., Ott, U., Hempel, H., Hackl, A., Wolf, K., Stark, R., \& Vaitl, D. (2007). Differential engagement of anterior cingulate and adjacent medial frontal cortex in adept meditators and non-meditators. Neuroscience letters, 421(1), 16-21.doi: 10.1016/j.neulet.2007.04.074 
Howard, G. S. (1980). Response-shift bias a problem in evaluating interventions with pre/post self-reports. Evaluation Review, 4(1), 93-106.doi: 10.1177/0193841X8000400105

Howdoes mindfulness-based cognitive therapy work? Behavior Research and Therapy,48,11051112.

Jo, Y., \& Bouffard, L. (2014). Stability of self-control and gender. Journal of Criminal Justice, 42, 356-365.

Klimecki, O. M., Leiberg, S., Lamm, C., \& Singer, T. (2013). Functional neural plasticity and associated changes in positive affect after compassion training. Cerebral Cortex, 23(7), 1552-1561. doi: 10.1093/cercor/bhs142

Kuyken, W., Watkins, E., Holden, E., White, K., Taylor, R. S., Byford, S., et al. (2010).

Lazar, S. W., Rosman, I. S., Vangel, M., Rao, V., Dusek, H., Benson, H., et al. (2003), November). Functional brain imaging of mindfulness and mantra-based meditation. Paper presented at the meeting of the Society for Neuroscience, New Orleans, LA

Lindman, H. R. (1974). Analysis of variance in complex experimental designs. W.H. Freeman \& Co.: London, UK.

Logie, K., \& Frewen, P. (2014). Self/Other Referential Processing Following

Longe, O., Maratos, F. A., Gilbert, P., Evans, G., Volker, F., Rockliff, H., \& Rippon, G. (2010). Having a word with yourself: Neural correlates of self-criticism and self-reassurance. Neuroimage, 49(2), 1849-1856 doi: 10.1016/j.neuroimage.2009.09.019

Marsh, A., Finger, E., Mitchell, D., Reid, M., Sims, C., Kosson, D., ... \& Blair, R. (2008). Reduced amygdala response to fearful expressions in children and adolescents with callous-unemotional traits and disruptive behavior disorders. American Journal of Psychiatry, 165(6), 712-720.doi: 10.1176/appi.ajp.2007.07071145

Masicampo, E. J., \& Baumeister, R. F. (2007). Relating mindfulness and self-regulatory processes. Psychological Inquiry, 18: 255-258.

McCord, J. (1991). Questioning the value of punishment. Social Problems, 38, 167-179. doi: 10.1525/sp.1991.38.2.03a00040

McCrory, E., De Brito, S. A., \& Viding, E. (2010). Research review: the neurobiology and genetics of maltreatment and adversity. Journal of Child Psychology and Psychiatry, 51(10), 1079-1095.

method biases in behavioral research: a critical review of the literature and recommended remedies. Journal of applied psychology, 88(5), 879.doi: 10.1037/0021-9010.88.5.879

Mindfulness and Loving-Kindness Meditation. Mindfulness, 1-10. doi: 10.1007/s12671-0140317-z

Moffitt, T. E. (1993). Adolescence-limited and life-course-persistent antisocial behavior: a developmental taxonomy. Psychological review, 100(4), 674. doi: 10.1037/0033295X.100.4.674

Murphy, C. M., Stosny, S., \& Morrel, T. M. (2005). Change in self-esteem and physical aggression during treatment for partner violent men. Journal of Family Violence, 20(4), 201-210. doi: 10.1007/s10896-005-5983-0 
Na, C., \& Paternoster, R. (2012). Can self-control change substantially over time? Rethinking the relationship between self- and social control. Criminology, 50: 427-462.

Nagin, D. S., \& Paternoster, R. (1993). Enduring individual differences and rational choice theories of crime. Law \& Society Review, 27: 467-496.

Neff, K. (2003b). Self-compassion: An alternative conceptualization of a healthy attitude toward oneself. Self and identity, 2(2), 85-101. doi: 10.1080/15298860309032

Neff, K. D. (2003a). The development and validation of a scale to measure self-compassion. Self and Identity, 2(3), 223-250. doi: 10.1080/15298860309027

Neff, K. D., \& Dahm, K. A. (in press). Self-Compassion: What it is, what it does, and how it relates to mindfulness. To appear in in M. Robinson, B. Meier \& B. Ostafin (Eds.) Mindfulness and Self-Regulation. New York: Springer.

Neff, K. D., \& Vonk, R. (2009). Self-Compassion Versus Global Self-Esteem: Two Different Ways of Relating to Oneself. Journal of personality, 77(1), 23-50. doi: 10.1111/j.14676494.2008.00537.x

Neff, K. D., Hseih, Y., \& Dejitthirat, K. (2005). Self-compassion, achievement goals, and coping with academic failure. Self and Identity, 4: 263-287.

Neff, K. D., Hsieh, Y. P., \& Dejitterat, K. (2005). Self-compassion, achievement goals, and coping with academic failure. Self and Identity, 4(3), 263-287. doi: 10.1080/13576500444000317

Netter, P., Hennig, J., Rohrmann, S., Wyhlidal, K., \& Hain-Hermann, M. (1998). Modification of experimentally induced aggression by temperament dimensions. Personality and individual differences, 25(5), 873-888. doi: 10.1016/S0191-8869(98)00070-1

Oser, C. (2006). The criminal offending-self-esteem nexus: Which version of the self-esteem theory is supported?The Prison Journal,86: 344-363.

Piquero , A. R., MacDonald, J., Dobrin, A., Daigle, L.E., Cullen, F. T., (2005). Self-control, violent offending, and homicide victimization: assessing the general theory of crime, Journal of Quantitative Criminology,21: 55-71.

Podsakoff, P. M., MacKenzie, S. B., Lee, J. Y., \& Podsakoff, N. P. (2003). Common

Raes, F., Pommier, E., Neff, K. D., \& Van Gucht, D. (2011). Construction and factorial

Robins, L. N. (1966). Deviant children grown up. Baltimore: Williams and Wilkins

Sampson, R J. \& Laub, J. H. (1993). Crime in the Making. Cambridge: Harvard University Press.

Schiffer, B., Pawliczek, C., Müller, B., Forsting, M., Gizewski, E., Leygraf, N., \& Hodgins, S. (2014). Neural mechanisms underlying cognitive control of men with lifelong antisocial behavior. Psychiatry Research: Neuroimaging.Doi 10.1016/j.pscychresns.2014.01.008

Siever, L. J., \& Davis. K. (1991). A psychobiological perspective on the personality disorders. American Journal of Psychiatry,148, 1647-1658.

Stosny, S. (1995). Treating attachment abuse: A compassion approach, Springer, New York.

Tow, P. M., \& Whitty, C. W. M. (1953). Personality changes after operations on the cingulate gyrus in man. Journal of neurology, neurosurgery, and psychiatry, 16(3), 186. 
validation of a short form of the self-compassion scale. Clinical psychology \& psychotherapy, 18(3), 250-255.

Vaughn, M. G., DeLisi, M., Beaver, K. M., Wright, J. P., \& Howard, M. O. (2007). Toward a psychopathology of self-control theory: the importance of narcissistic traits. Behavioral sciences \& the law, 25(6), 803-821. doi: 10.1002/bsl.789

Vissing, Y. M., Straus, M. A., Gelles, R. J., \& Harrop, J. W. (1991). Verbal aggression by parents and psychosocial problems of children. Child Abuse \& Neglect, 15(3), 223-238. doi: 10.1016/0145-2134(91)90067-N

Wang, D. J., Rao, H., Korczykowski, M., Wintering, N., Pluta, J., Khalsa, D. S., \& Newberg, A. B. (2011). Cerebral blood flow changes associated with different meditation practices and perceived depth of meditation. Psychiatry Research: Neuroimaging, 191(1), 60-67. doi: 10.1016/j.pscychresns.2010.09.011

Watson, David, Lee A. Clark, and Auke Tellegen. "Development and validation of brief measures of positive and negative affect: the PANAS scales." Journal of personality and social psychology 54.6 (1988): 1063.

Watson, J., Solomon, A. L., La Vigne, N. G., Travis, J., Funches, M., \& Parthasarathy, B. (2004). Portrait of Prisoner Reentry in Texas. Urban League 1-124

Web, J. B., \&Forman, J. B., (2013) Evaluating the indirect effect of self-compassion on binge eating severity through cognitive-affective self-regulatory pathways. Eating Behaviors. 14: 224-228 doi: 10.1016/j. eatbeh.2012 12.005

Weiler, B. L., \& Wisdom, C. S. (1996). Psychopathy and violent behavior in abused and neglected young adults. Criminal Behavior and Mental Health, 6, 253-271.

Widom, C. S. (1989). Child abuse, neglect, and adult behavior: research design and findings on criminality, violence, and child abuse. American Journal of Orthopsychiatry, 59(3), 355. doi: 10.1111/j.1939-0025.1989.tb01671.x

Woldgabreal, Y., Day, A., \& Ward, T. (2014). The community-based supervision of offenders from a positive psychology perspective. Aggression and Violent Behavior, 19(1), 3241.doi:10.1016/j.avb.2013.12.001

Yang, Y., \& Raine, A. (2009). Prefrontal structural and functional brain imaging findings in antisocial, violent, and psychopathic individuals: a meta-analysis. Psychiatry Research: Neuroimaging, 174(2), 81-88. doi: 10.1016/j.pscychresns.2009.03.012 
Table 1: Means and Standard Deviations for Variables

Mean

Std. Error

Hostility Scale Time 1

Self-Control

Low Self-Control Group

73.22

3.9

Medium Self-Control Group

68.71

4.5

High Self-Control Group

65.14

5.3

Self-Compassion

Low Self-Compassion Group

82.5

4.3

Medium Self-Compassion Group

High Self-Compassion Group

66

5.3

58.6

4.23

Hostility Time 2

Self-Control

Low Self-Control

Medium Self-Control

High Self-Control

85

70.51

4.75

69.6

5.52

6.4

Self-Compassion*Self-Control*Hostility Time 2

Low Self-Compassion* Low Self-Control 117

Low Self-Compassion* Medium Self-Control

Low Self-Compassion* High Self-Control

68.8

7.5

82.7

10.05

Medium Self-Compassion*Low Self-Control

70.2

6.57

Medium Self-Compassion*Medium Self-Control

71.71

5.57

Medium Self-Compassion* High Self-Control

66.5

8.5

High Self-Compassion* Low Self-Control

67.6

15.89

High Self-Compassion*Medium Self-Control

71

8.26

High Self-Compassion* High Self-Control

59.67

10.05

6

Table 2

\begin{tabular}{|c|c|c|c|c|}
\hline Ho & ity Pre & Hostility Post & Self-Compassion & Self-Control \\
\hline Hostility Pre & 1 & & & \\
\hline Hostility Post & $.28^{*}$ & 1 & & \\
\hline Self-Compassion & $-.56 * *$ & $-.48 * *$ & 1 & \\
\hline Self-Control & $.28 *$ & $.33 * *$ & $-.27 *$ & 1 \\
\hline
\end{tabular}

$* \mathrm{p}<.05 \quad * * \mathrm{p}<.01$ 